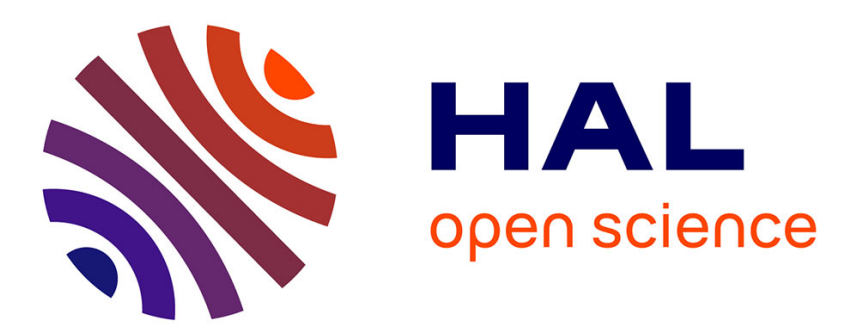

\title{
Étude de la susceptibilité du composé C8K à basse température par résonance paramagnétique électronique
}

\author{
J. Poitrenaud
}

\section{To cite this version:}

J. Poitrenaud. Étude de la susceptibilité du composé C8K à basse température par résonance paramagnétique électronique. Revue de Physique Appliquée, 1970, 5 (2), pp.275-281. 10.1051/rphysap:0197000502027500 . jpa-00243388

\section{HAL Id: jpa-00243388 https://hal.science/jpa-00243388}

Submitted on 1 Jan 1970

HAL is a multi-disciplinary open access archive for the deposit and dissemination of scientific research documents, whether they are published or not. The documents may come from teaching and research institutions in France or abroad, or from public or private research centers.
L'archive ouverte pluridisciplinaire HAL, est destinée au dépôt et à la diffusion de documents scientifiques de niveau recherche, publiés ou non, émanant des établissements d'enseignement et de recherche français ou étrangers, des laboratoires publics ou privés. 


\title{
ÉTUDE DE LA SUSCEPTIBILITÉ DU COMPOSÉ $\mathrm{C}_{8} \mathrm{~K}$ A BASSE TEMPÉRATURE PAR RÉSONANCE PARAMAgNÉTIQUe ÉlectroniQue
}

\author{
par J. POITRENAUD
}

Service de Physique du Solide et de Résonance Magnétique,

Centre d'Etudes Nucléaires de Saclay, B. P. no 2, 91, Gif-sur-Yvette

(Reçu le 31 octobre 1969)

\begin{abstract}
Résumé. - La susceptibilité paramagnétique du composé $\mathrm{C}_{8} \mathrm{~K}$ a été mesurée en fonction de la température de $300^{\circ} \mathrm{K}$ à $0,4^{\circ} \mathrm{K}$ en étudiant la résonance des électrons de conduction. Ces mesures permettent de conclure que $\mathrm{C}_{8} \mathrm{~K}$ a le comportement d'un métal. Aucune transition à l'état supraconducteur n'a pu être mise en évidence dans les différents échantillons utilisés refroidis jusqu'à $0,3{ }^{\circ} \mathrm{K}$. L'appareillage utilisé - spectromètre à $300 \mathrm{MHz}$, cryostat à ${ }^{3} \mathrm{He}$ et pont $\mathrm{B}$. F. de détection de la supraconductivité - est décrit en détail.

Abstract. - The paramagnetic susceptibility of the $\mathrm{C}_{8} \mathrm{~K}$ compound has been measured as a function of temperature from $300{ }^{\circ} \mathrm{K}$ to $0.4^{\circ} \mathrm{K}$ by studying the intensity of the conduction electron spin resonance. It is found that $\mathrm{C}_{8} \mathrm{~K}$ has a metallic behaviour. Different samples of $\mathrm{C}_{8} \mathrm{~K}$ have been investigated for superconductivity down to $0.3{ }^{\circ} \mathrm{K}$, but no transition has been found. The apparatus - a $300 \mathrm{MHz}$ spectrometer, a ${ }^{3} \mathrm{He}$ cryostat and a low frequency superconductivity detection bridge - is described in detail.
\end{abstract}

I. Introduction. - La susceptibilité paramagnétique d'un métal normal est indépendante de la température alors que celle d'un métal supraconducteur, comme l'a calculé Yosida [1], tend exponentiellement vers 0 lorsque la température approche $0{ }^{\circ} \mathrm{K}$. Cependant de nombreux expérimentateurs ont mesuré des déplacements de Knight de métaux supraconducteurs différents de 0 à $0 \circ \mathrm{K}$. Une explication a été proposée qui semble expliquer ce désaccord [2]. Cependant la mesure du déplacement de Knight ne paraît pas être la plus appropriée à la détermination de la susceptibilité paramagnétique car elle fait intervenir de nombreux autres facteurs, ce qui rend délicate l'interprétation des résultats, alors que, ainsi que l'ont montré Schumacher et Slichter [3], la susceptibilité paramagnétique d'un métal peut être mesurée de façon très précise en étudiant l'intensité de la résonance des électrons de conduction dans le cas où la raie est étroite. C'est pourquoi il serait intéressant d'observer la résonance électronique d'un composé dans son état supraconducteur. Cette résonance est en principe observable si elle est visible dans le composé à l'état normal. Kaplan [4] a envisagé le cas des supraconducteurs en couches minces, de Gennes [5] celui des supraconducteurs massifs de $2^{\mathrm{e}}$ espèce. De Gennes montre que l'élargissement inhomogène dû à la présence des lignes de tourbillon est fortement diminué par le mouvement des porteurs.

Il se trouve que le composé $\mathrm{C}_{8} \mathrm{~K}$ semble être un des seuls composés connus qui obéissent aux conditions requises [6]. $\mathrm{C}_{8} \mathrm{~K}$ fait partie des composés de type intercalaire obtenus en mettant en contact le graphite avec un métal alcalin gazeux, $\mathrm{K}, \mathrm{Rb}$ ou $\mathrm{Cs}$. Leur formule générale est $\mathrm{C}_{n} \mathrm{M}$ où $n$ peut être égal à 8 (composé doré), 24 (composé bleu), 36, 48, 60. L'examen de ces composés au moyen des rayons $X$ [7] a montré que les atomes de métal se placent dans des plans intercalés entre les plans des carbones. La bande de conduction vide du graphite commence à se remplir. Les mesures [8] de la variation de la résistivité et de la puissance thermoélectrique avec la concentration en métal alcalin montrent, dans le cas du potassium, que le transfert d'électrons rapporté à un atome de potassium est compris entre 0,1 et 0,4 .

La résonance électronique de $\mathrm{C}_{8} \mathrm{~K}, \mathrm{C}_{24} \mathrm{~K}$ et $\mathrm{C}_{24} \mathrm{Rb}$ a été examinée [9] à $300^{\circ} \mathrm{K}$ et $77^{\circ} \mathrm{K}$ à l'aide d'un spectromètre à $3 \mathrm{~cm}$. Voici les résultats en ce qui concerne $\mathrm{C}_{8} \mathrm{~K}$ :

\begin{tabular}{|c|c|c|c|c|c|c|}
\hline \multicolumn{2}{|c|}{$g_{\|}$} & \multicolumn{2}{|c|}{$g_{\perp}$} & \multicolumn{2}{|c|}{$\Delta H$ en gauss } & intensité $300^{\circ} \mathrm{K}$ \\
\hline $300 \% \mathrm{~K}$ & $77^{\circ} \mathrm{K}$ & $300^{\circ} \mathrm{K}$ & $77^{\circ} \mathrm{K}$ & $300^{\circ} \mathrm{K}$ & $77^{\circ} \mathrm{K}$ & intensité $77^{\circ} \mathrm{K}$ \\
\hline - & - & - & - & 一 & 一 & - \\
\hline 2,0023 & 2,0022 & 2,0037 & 2,0031 & 14,3 & 5,6 & 3,4 \\
\hline $\pm 0,0002$ & $\pm 0,0003$ & $\pm 0,0002$ & $\pm 0,0002$ & $\pm \quad 0,2$ & $\pm 0,1$ & \\
\hline
\end{tabular}


Un groupe de chercheurs [10] a étudié la supraconductivité de ces composés. Elle n'a pu être mise en évidence que dans $\mathrm{C}_{8} \mathrm{M}$. La température de transition la plus élevée a été observée pour $\mathrm{C}_{8} \mathrm{~K}$. Les valeurs de la température de transition $T_{\mathrm{c}}$ et du champ critique $H_{\mathrm{c} 2}$ sont données dans le tableau suivant (c est l'axe perpendiculaire aux plans des carbones).

\begin{tabular}{lccc}
\multicolumn{4}{c}{ TABLEAU II } \\
& $T_{\mathrm{c}}$ & $H_{\mathrm{c} 2}\left(H_{\| \mathrm{c}}\right)$ & $H_{\mathrm{c} 2}\left(H_{\perp \mathrm{c}}\right)$ \\
& - & - & - \\
& - & - & - \\
$C_{8} \mathrm{~K}$, K en excès & $0,55^{\circ} \mathrm{K}$ & $730 \mathrm{G}$ & $160 \mathrm{G}$ \\
$C_{8} \mathrm{~K}$ Stoech & $0,39^{\circ} \mathrm{K}$ & $250 \mathrm{G}$ & $25 \mathrm{G}$
\end{tabular}

Afin de pouvoir étudier en fonction de la température la susceptibilité paramagnétique de ce composé dans son état normal et dans son état supraconducteur, les données précédentes imposent l'observation de la résonance à bas champ et à des températures pouvant diminuer jusqu'à $0,3^{\circ} \mathrm{K}$. Un spectromètre à $300 \mathrm{MHz}$ et un cryostat à ${ }^{3} \mathrm{He}$ ont été construits permettant d'étudier la résonance dans un champ de $100 \mathrm{G}$ et à des températures allant jusqu'à $0,32^{\circ} \mathrm{K}$. De plus comme aucune modification de la raie de résonance n'est apparue en baissant la température, un pont basse fréquence de détection de la supraconductivité a été construit. Nous n'avons pu mettre de transition en évidence dans aucun échantillon étudié. C'est pourquoi nous décrirons en détail l'appareillage utilisé afin de bien préciser les conditions dans lesquelles cette expérience a été réalisée. Nous donnerons les résultats déduits de l'observation de la résonance de $\mathrm{C}_{8} \mathrm{~K}$ à l'état normal.

II. Cryostat à ${ }^{3}$ He. - 1) Description. - Le schéma de principe du cryostat est reproduit figure 1.

Ce cryostat est du type classique à un seul cycle de ${ }^{3} \mathrm{He}$. Le choix des matériaux, la forme et les diamètres de pompage ont été imposés par les conditions suivantes: premièrement, l'échantillon à refroidir se présente sous forme de lamelles qui s'oxydant très rapidement à l'air sont conservées sous huile dans des ampoules de pyrex scellées sous faible pression de ${ }^{3} \mathrm{He}$. Ces ampoules ont $5 \mathrm{~mm}$ de diamètre extérieur et en moyenne $5 \mathrm{~cm}$ de hauteur. Deuxièmement nous voulions pouvoir changer facilement d'échantillon à basse température $\left(4,2^{\circ} \mathrm{K}\right)$. Troisièmement afin d'éviter des pertes par effet Joule et conduction thermique trop importantes, il est nécessaire de placer l'hélice de la cavité résonnante $\uparrow$ à l'extérieur du bain de ${ }^{3} \mathrm{He}$. La queue du cryostat (2) doit donc être construite avec un matériau non conducteur et ne donnant aucun signal de résonance électronique. Il faut noter que travaillant à $300 \mathrm{MHz}$, cette condition est assez contraignante car les signaux à $g$ très différent de 2 peuvent gêner l'observation du signal à $g=2$. Par exemple le pyrex donne à $g=4(H=50 \mathrm{G})$ un signal très important

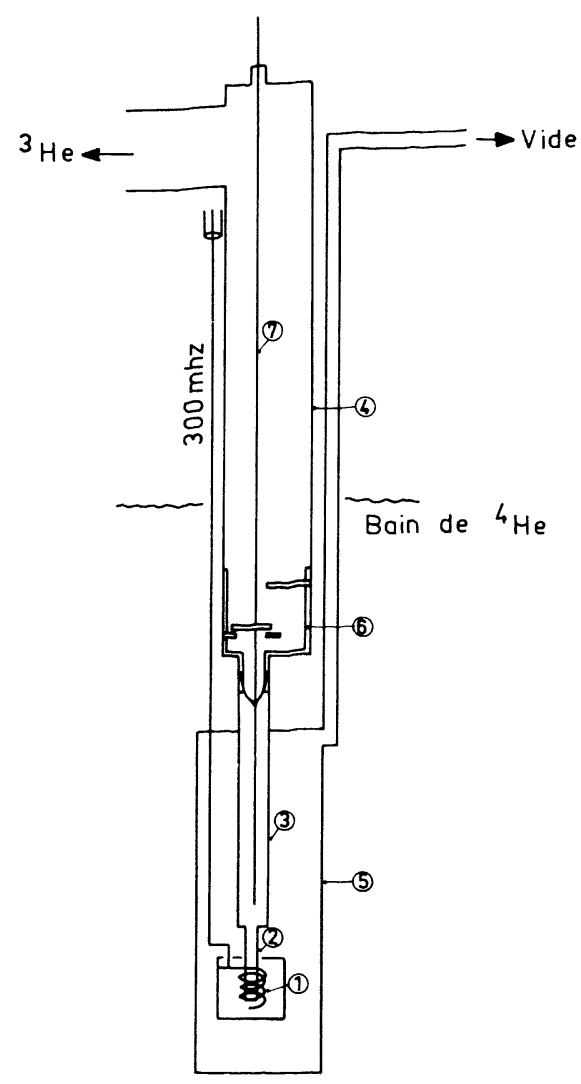

FIg. 1. - Schéma du cryostat à 3 He.

qui perturbe la ligne de base autour du champ d'observation de $100 \mathrm{G}$. De plus il donne ainsi que le quartz un signal à $g=2$. Ces deux matériaux ne conviennent donc pas. Par contre nous avons vérifié que le kel $F$, l'araldite, le nylon, l'épibond $100 \mathrm{~A}$ ne donnent pas de signaux à $g=2$. Le nylon a été choisi pour ses propriétés mécaniques. La chambre d'expérience (2) est donc constituée par un tube de nylon de diamètre $7 \times 9 \mathrm{~mm}$, de hauteur $70 \mathrm{~mm}$. La jonction avec la ligne de pompage de ${ }^{3} \mathrm{He}$ se fait par l'intermédiaire d'une pièce spéciale en laiton. La ligne de pompage est en acier inoxydable très mince $1 / 10 \mathrm{~mm}$ dans la partie froide (3) jusqu'à $1,2{ }^{\circ} \mathrm{K}$; son diamètre est alors $12 \mathrm{~mm}$ il passe à $30 \mathrm{~mm}$ dans la partie supérieure (4). Le pompage est réalisé à l'aide d'une pompe secondaire à vapeur d'huile de débit $120 \mathrm{1} / \mathrm{s}$. La partie froide est isolée du bain de ${ }^{4} \mathrm{He}$ par une enceinte vide (5) dans laquelle est placée la cavité résonnante. Le câble coaxial qui assure le couplage de la cavité au générateur est rendu étanche au niveau de l'entrée dans la cavité au moyen d'un scellement d'épibond 100 A [11].

Les deux premières conditions imposent que la ligne de pompage ne comporte aucun coude. Pour éviter que l'énergie rayonnée le long du tube ne se dissipe dans le bain de ${ }^{3} \mathrm{He}$ on intercale un "piège à radiations " (6) constitué par une pièce de cuivre en bon contact thermique avec le bain de ${ }^{4} \mathrm{He}$ à $1,2{ }^{\circ} \mathrm{K}$. Cette pièce comporte des écrans en chicane, l'un d'eux percé d'un trou circulaire permet le passage de l'échan- 
tillon, la tige porte échantillon comportant une rondelle de cuivre qui ferme cette ouverture lorsque l'échantillon est en place. La tige porte échantillon ? est un tube d'acier inoxydable de $1 \times 1,5 \mathrm{~mm}$ de diamètre à l'extrémité duquel est fixé un tube de cuivre de diamètre $5 \times 6 \mathrm{~mm}$. L'ampoule contenant l'échantillon est fixée dans ce tube. Les pertes par conduction sont rendues négligeables en assurant le contact thermique entre la tige et le bain de ${ }^{4} \mathrm{He}$ à $1,2{ }^{\circ} \mathrm{K}$ par des balais de cuivre soudés sur la tige et glissant sur la pièce $n^{0}$ (6).

Nous disposons de 3 litres de ${ }^{3} \mathrm{He}$ à la pression atmosphérique. Les pertes thermiques étant de $10 \mu \mathrm{W}$, l'autonomie du cryostat est de plusieurs heures.

2) Mesure de la température. - Les températures sont mesurées à l'aide de résistances au carbone plongées dans le bain de ${ }^{3} \mathrm{He}$. Ces thermomètres ont été étalonnés en mesurant la variation de l'intensité $I$ d'un signal de résonance paramagnétique électronique d'un cristal de double nitrate de lanthane et de magnésium (LMN) dopé à $1 \%$ de néodyme. La résonance est observée avec un faible niveau de puissance haute fréquence $\left(10^{-2} \mu \mathrm{W}\right)$. I varie comme $1 / T$. On dispose d'un contrôle supplémentaire avec la mesure de $T$ en fonction de la pression de vapeur de ${ }^{3} \mathrm{He}$. Cette détermination n'a de sens que jusqu'à une pression de vapeur de l'ordre de $70 \mu$ de mercure $\left(T \sim 0,45^{\circ} \mathrm{K}\right)$. On peut ainsi tracer $I=f(T)$ en coordonnées logarithmiques (Fig. 2) et vérifier que l'on obtient une

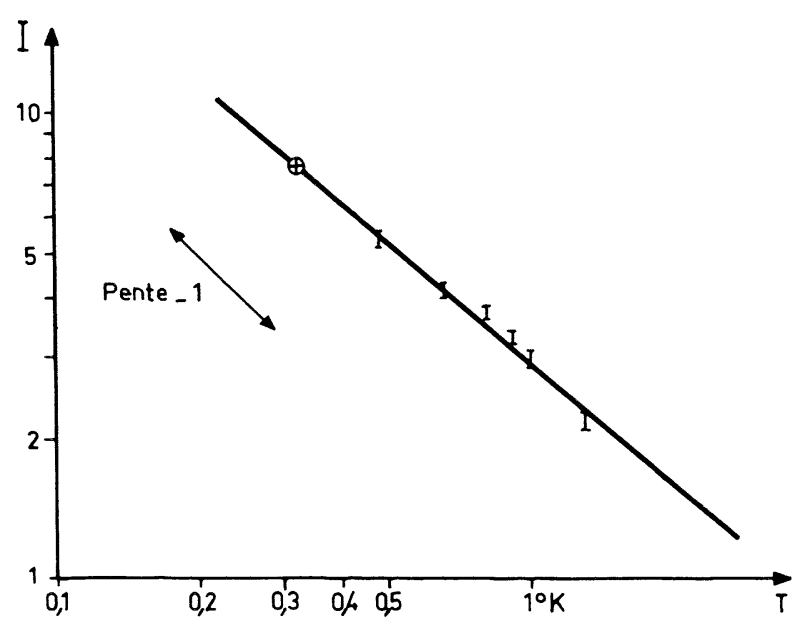

Fig. 2. - Variation de l'intensité du signal de résonance donné par un cristal de LMN à $1 \%$ de Nd en fonction de la température mesurée par l'intermédiaire de la pression de vapeur de ${ }^{3} \mathrm{He}$ pour $T>0,45^{\circ} \mathrm{K}$. Pour $T<0,45^{\circ} \mathrm{K}$, cette droite permet de déduire $T$ de la mesure de $I$ (croix entourée d'un cercle).

droite de pente -1 . A $T<0,45^{\circ} \mathrm{K}$ les pressions mesurées en haut de la colonne de pompage sont très différentes de celles au point froid et les corrections [12] à y apporter trop incertaines. Dans ce cas on mesure $I$ et on en déduit $T .0,32^{\circ} \mathrm{K}$ est la température minimale obtenue.
Il faut noter cependant qu'un certain nombre de précautions doit être observé. En effet à cause de la forme de l'échantillon le bain de ${ }^{3} \mathrm{He}$ se présente comme une colonne assez haute (de l'ordre de $10 \mathrm{~cm}$ ). Lorsque l'on commence à pomper sur l'hélium liquéfié à $1,2^{\circ} \mathrm{K}$, la température en haut du bain baisse instantanément mais le temps mis pour que la température du bain s'équilibre n'est pas négligeable. Wheatley [13] a fait des études systématiques des transferts de chaleur à basse température. Le temps $\tau$ mis pour que l'équilibre de température s'établisse le long d'un matériau de longueur $l$, de section $s$, de coefficient de conductivité thermique $\lambda$ et de capacité calorifique $C_{v}$ est

$$
\tau \sim \frac{C_{v} l^{2} s}{\lambda s},
$$

or ${ }^{3} \mathrm{He}$ a une très grande capacité calorifique $0,12 \mathrm{~J} / \mathrm{cm}^{3} \mathrm{o}$ à $1,2{ }^{\circ} \mathrm{K}$ et les matériaux du cryostat (nylon, pyrex ainsi que ${ }^{3} \mathrm{He}$ lui-même) [14] ont de faibles coefficients de conduction thermique, de sorte que si on ne prend pas de précautions $\tau$ peut être exagérément long, de l'ordre de quelques heures. Les matériaux permettant les transferts de chaleur les plus rapides sont le cuivre et le quartz monocristallin. C'est la raison pour laquelle l'ampoule contenant l'échantillon est placée dans un tube de cuivre. Ce tube ne va pas jusqu'au fond du bain car il ne peut pénétrer dans la cavité résonnante sous peine de diminuer de façon catastrophique son facteur de qualité. La figure 3 montre la variation de la température en haut (1) et en bas (2) du bain en fonction du temps. $\tau$ est alors de l'ordre de $30 \mathrm{mn}$.

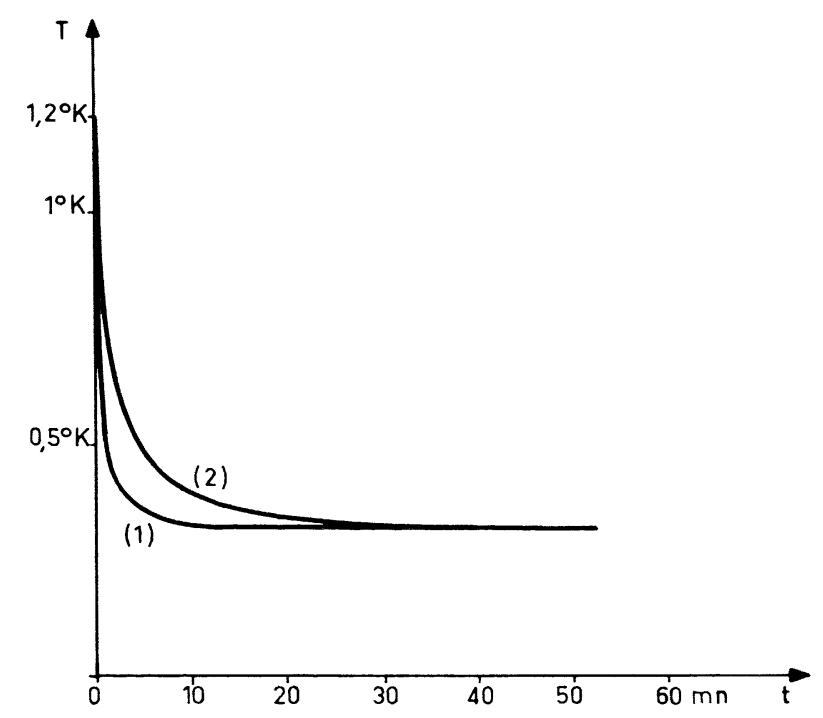

FIG. 3. - Variation de la valeur des températures en haut (1) et en bas (2) du bain de ${ }^{3} \mathrm{He}$ en fonction du temps (le temps $t=0$ représente le début du pompage).

D'autre part, l'apport de chaleur par pertes diélectriques lorsqu'on branche la haute fréquence provoque une élévation de la température. Enfin la modulation basse fréquence du champ magnétique statique peut 
produire un échauffement du bain si la fréquence est trop élevée. Cet effet est provoqué sans doute par les vibrations.

Les résultats des mesures de températures en fonction 'de la puissance haute fréquence et de la fréquence de la modulation appliquées sont les suivants

\section{TABLEAU III}

$\begin{array}{rcc}\text { pce hf } & T(\Omega=40 \mathrm{~Hz}) & T(\Omega=300 \mathrm{~Hz}) \\ - & - & - \\ 100 \mathrm{~mW} & 0,60{ }^{\circ} \mathrm{K} & \\ 10 \mathrm{~mW} & 0,40{ }^{\circ} \mathrm{K} & \\ 1 \mathrm{~mW} & 0,32{ }^{\circ} \mathrm{K} & 0,40{ }^{\circ} \mathrm{K}\end{array}$

III. Mesure de la susceptibilité. - 1) LE SPECTROMÈTRE A $300 \mathrm{MHz}$. - Le spectromètre qui a été construit avec la collaboration du Dr O. Lumpkin est du type superhétérodyne à réflexion. Le schéma de principe est représenté sur la figure 4. La cavité résonnante s'inspire des modèles utilisés à fréquence plus élevée. C'est une cavité coaxiale $\lambda / 4$. Mais à $300 \mathrm{MHz}$

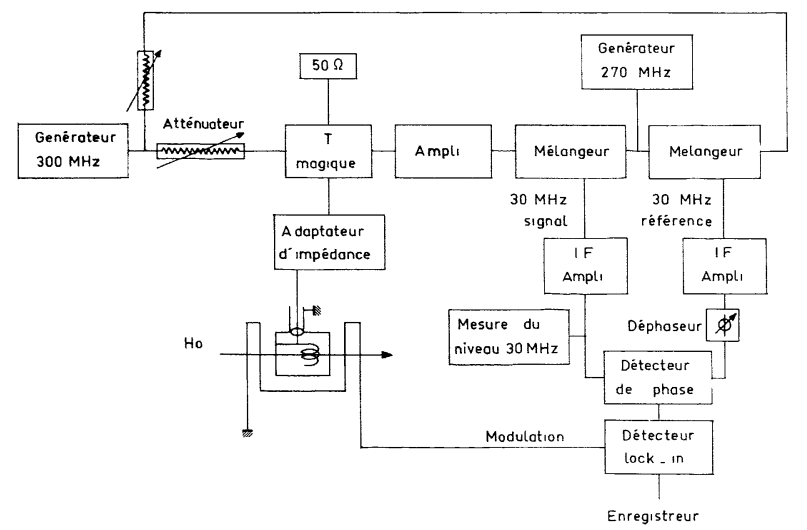

Fig. 4. - Schéma du spectromètre à $300 \mathrm{MHz}$.

la longueur d'onde est $\lambda=1 \mathrm{~m}$. Aussi le conducteur central est-il enroulé en hélice, ce qui permet de ralentir l'onde qui se propage. Les caractéristiques de ces cavités sont décrites dans la référence [15]. Le facteur de qualité $Q_{0}$ d'une telle cavité non chargée est :

$$
Q_{0}=50 \mathrm{D} \sqrt{f_{0}}
$$

$D$ : diamètre du blindage exprimé en inch, $f_{0}$ : fréquence de résonance. On trouve $Q_{0}$ de l'ordre de 900 .

L'hélice et le blindage de la cavité sont dorés afin de supprimer les signaux parasites. Le couplage avec le générateur se fait par l'intermédiaire d'un câble coaxial de $50 \Omega$ directement soudé sur l'hélice. A la résonance l'impédance le long de l'hélice varie de 0 à $\infty$. L'adaptation à $50 \Omega$ est réalisée d'abord en plaçant la soudure au mieux au voisinage de $50 \Omega$ puis à l'aide d'un adaptateur d'impédance commercial.

La sensibilité d'un spectromètre peut être mesurée en considérant le nombre minimum de spins détectables soit [15] [16] :

$$
N_{\text {min }}=\left(\frac{F k T_{0} \Delta v}{2 P_{0}}\right)^{1 / 2} \frac{v_{\text {eff }}}{Q_{0}} \frac{3 k T}{\mu^{2}} \frac{\Delta H}{H},
$$

où $F$ est la figure de bruit de l'ensemble, $\Delta v$ la largeur de bande du détecteur, $T_{0}$ sa température, $P_{0}$ la puissance haute fréquence incidente, $v_{\text {eff }}$ le volume utile de la cavité, $\mu$ le moment magnétique de l'électron, $\Delta H$ la largeur de raie, $H$ le champ magnétique appliqué et $T$ la température de l'échantillon. Si on veut comparer la sensibilité d'un tel spectromètre à celle d'un appareil fonctionnant à $9000 \mathrm{MHz}$, on constate que $N_{\min }$ est proportionnel à

$$
F^{1 / 2} \times \frac{1}{Q_{0}} \times \frac{1}{H}
$$

or la figure de bruit est déterminée principalement par celle de l'amplificateur et du mélangeur. Aux fréquences centimétriques elle est de l'ordre de $14 \mathrm{db}$ alors qu'à $300 \mathrm{MHz}$ on arrive à fabriquer des appareils moins bruyants ayant une figure de bruit de $6 \mathrm{db}$. L'effet dû à la diminution de $Q$ et surtout à la diminution du champ magnétique par un facteur 30 est en partie compensé par la possibilité d'introduire des échantillons plus gros et dans le cas des métaux par l'augmentation de l'épaisseur de peau dans un rapport égal à la racine du rapport des fréquences soit 5,5. Donc en ce qui concerne les métaux la sensibilité d'un spectromètre à $300 \mathrm{MHz}$ est à peu près équivalente à celle d'un spectromètre à fréquence plus élevée.

Ce spectromètre a été testé à température ordinaire avec $1,5 \mathrm{mg}$ de diphényl-picryl-hydrazyl soit $2 \times 10^{18}$ spins. Le rapport de l'intensité du signal à celle du bruit est 500 pour $P_{0}=1 \mathrm{~mW} \cdot N_{\text {min }}$ expérimental est donc de l'ordre de $4 \times 10^{15}$ spins à $300^{\circ} \mathrm{K}$. La formule ci-dessus donnerait $10^{15}$ spins.

Dans le cas d'un métal la formule donnant $N_{\text {min }}$ doit être modifiée car uniquement la fraction $\mu H / E_{F}$ des électrons de conduction d'énergie voisine de l'énergie de Fermi $E_{F}$ participe à la résonance. On trouve :

$$
N_{\min }=\frac{F k T_{0} \Delta v^{1 / 2}}{2 P_{0}} \frac{v_{\text {eff }}}{Q_{0}} 3 \frac{E_{F}}{\mu^{2}} \frac{\Delta H}{H},
$$

qui ne dépend de la température que par l'intermédiaire de la largeur de raie. On trouve pour $\mathrm{C}_{8} \mathrm{~K}$ à température ordinaire $N_{\min } \sim 10^{17}$ spins pour une puissance haute fréquence de $1 \mathrm{~mW}$.

2) RÉSultats. - Nous avons examiné les signaux donnés par la résonance des électrons de conduction de $\mathrm{C}_{8} \mathrm{~K}$ de $300^{\circ} \mathrm{K}$ à $1,2^{\circ} \mathrm{K}$. Les figures 5 , 6 et 7 montrent les signaux observés avec une puissance de $100 \mathrm{~mW}$ pour un échantillon constitué par deux plaquettes de $12 \times 2,5 \mathrm{~mm}^{2}$ et d'épaisseur $100 \mu$. L'intensité $S_{T}$ de la raie observée à la température $T$, sa largeur $\Delta H$, son rapport d'asymétrie [18] $A / B$ sont donnés en fonction de la température dans le tableau IV. 


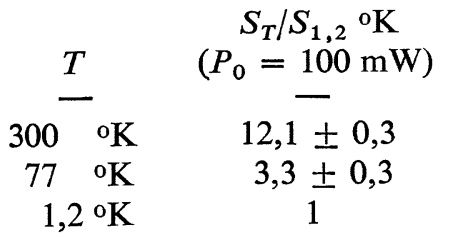

TABLeAU IV

\begin{tabular}{cccc}
$A / B$ & \multicolumn{3}{c}{$\delta_{\mu}$} \\
- & $\rho_{\Omega \times \mathrm{cm}}$ & $300 \mathrm{MHz}$ & $N_{T} / N_{1,2}{ }^{\circ} \mathrm{K}$ \\
$1,8 \pm 0,2$ & $5,5 \times 10^{-5}$ & -21 & 10,5 \\
$2,1 \pm 0,1$ & $6 \times 10^{-6}$ & 7 & 3,5 \\
$2,1 \pm 0,4$ & $5 \times 10^{-7}$ & 2 & 1
\end{tabular}

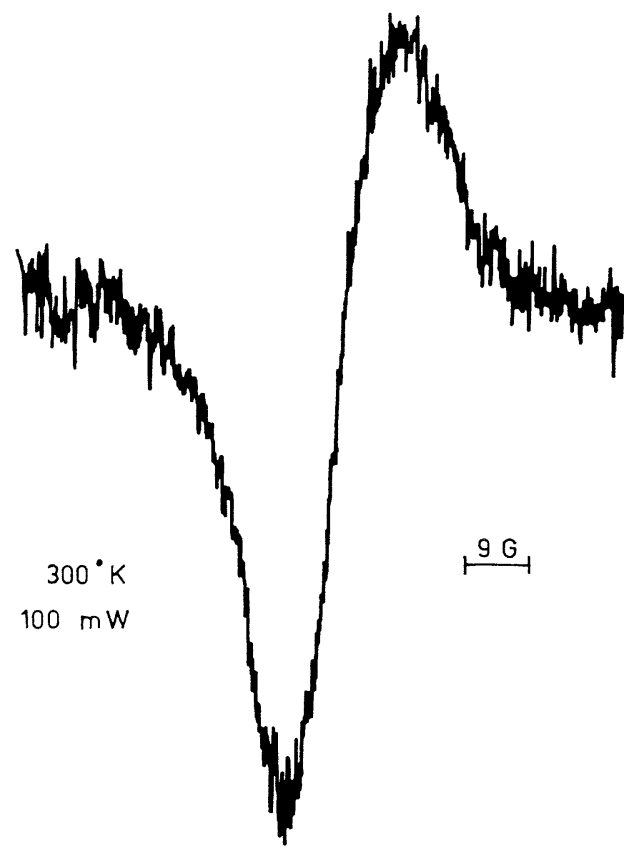

FIG. 5. - Signal dû à la résonance des électrons de conduction de $\mathrm{C}_{8} \mathrm{~K}$ à $300^{\circ} \mathrm{K}$.

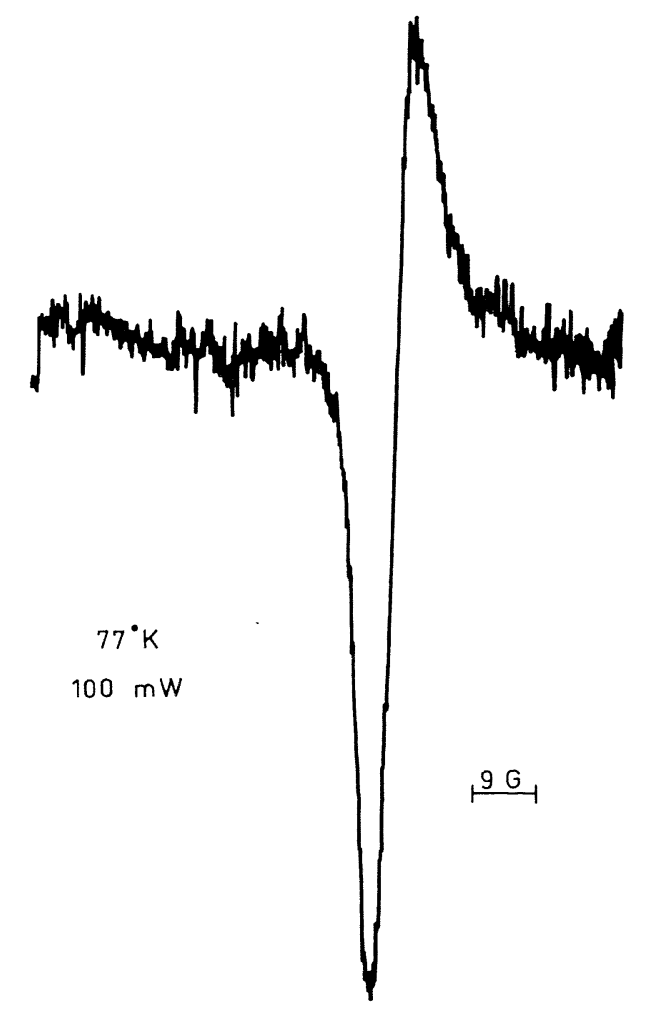

Fig. 6. - Signal dû à la résonance des électrons de conduction de $\mathrm{C}_{8} \mathrm{~K}$ à $77^{\circ} \mathrm{K}$.

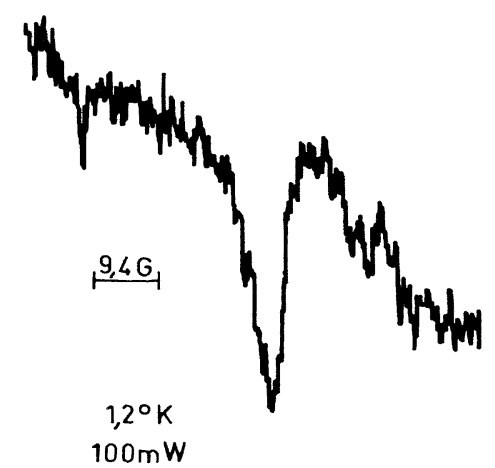

FIG. 7. - Signal dû à la résonance des électrons de conduction de $\mathrm{C}_{8} \mathrm{~K}$ à $1,2{ }^{\circ} \mathrm{K}$.

Nous y avons inscrit également les valeurs de la résistivité mesurées récemment [19] pour un courant circulant dans le sens de la plaquette ainsi que les valeurs de l'épaisseur de peau $\delta$ et celles relatives du nombre de spins $N$ contenus dans $\delta$ que l'on peut en déduire.

Les raies ont la forme dysonienne caractéristique d'un conducteur [18]. A $300^{\circ} \mathrm{K}$ l'épaisseur de peau $\delta=21 \mu$ n'est pas très petite par rapport à l'épaisseur $d$ de l'échantillon et le rapport d'asymétrie est compris entre $1(d \ll \delta)$ et $2,7(d \gg \delta)$. Quand la température diminue $A / B$ augmente. Pour étudier la variation" de l'intensité du signal il faut tenir compte qu'entre $300{ }^{\circ} \mathrm{K}$ et $77^{\circ} \mathrm{K}$ le $Q$ de la cavité est multiplié par 2 et la largeur de raie divisée par 2,5. On constate alors que la variation de l'amplitude du signal avec la température est entièrement expliquée par la variation du nombre de spins. Il semble donc, qu'à la précision des mesures près, $\mathrm{C}_{8} \mathrm{~K}$ ait le comportement d'un métal avec une susceptibilité constante en fonction de la température.

Si à partir de $1,2^{\circ} \mathrm{K}$ la température diminue jusqu'à $0,40{ }^{\circ} \mathrm{K}$, on ne constate aucune variation ni dans la forme, ni dans l'intensité de la raie. En principe une transition aurait dû dérégler le spectromètre. Ceci nous a amenés à reprendre l'étude de la supraconductivité des échantillons.

IV. Détection de la supraconductivité. - Cet appareillage consiste à mettre en évidence la variation du flux à travers une bobine entourant l'échantillon au moment où celui-ci devient supraconducteur. Le schéma de l'appareil est représenté figure 8 .

La bobine d'émission crée un champ magnétique oscillant. La bobine de réception d'axe parallèle est 


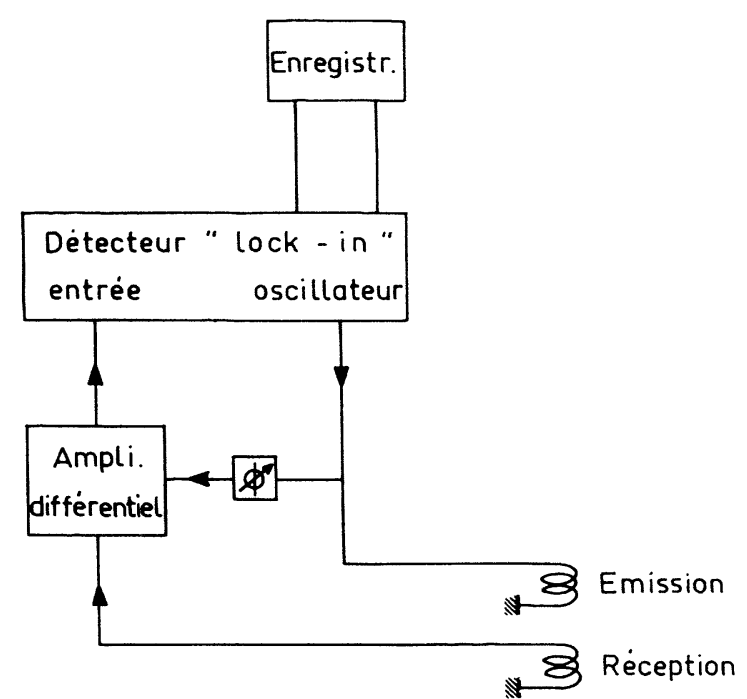

Fig. 8. - Schéma du pont B. F. de détection de la supraconductivité.

constituée par un grand nombre de spires. Par l'intermédiaire d'un amplificateur différentiel la tension recueillie aux bornes de la bobine réceptrice est compensée exactement par une fraction de la tension alimentant la bobine d'émission, tension ajustable tant en amplitude qu'en phase. Ceci évite de saturer le détecteur lock in et permet d'utiliser sa sensibilité maximale.

La variation de signal à la transition est :

$$
e=n H_{m} \Omega s \eta G
$$

où $H_{m}$ est l'intensité du champ magnétique oscillant créé par la bobine d'émission, $\Omega$ sa pulsation, $s$ la section de la bobine réceptrice, $n$ son nombre de spires, $\eta$ son coefficient de remplissage, $G$ le gain de l'amplificateur.

Il est évidemment intéressant d'avoir $\Omega, n$ et $\eta$ le plus grand possible. En plaçant la bobine de réception dans le bain de ${ }^{3} \mathrm{He}$ autour de l'ampoule contenant l'échantillon on arrive au mieux à $\eta=1 / 10$. $n$ est imposé alors par les dimensions respectives de l'ampoule et du cryostat; en utilisant du fil de $5 / 100 \mathrm{~mm}$ on peut réaliser un nombre de spires de l'ordre de 7000 . On ne peut choisir $\Omega$ trop grand sous peine d'échauffer le bain de ${ }^{3} \mathrm{He}$ comme on l'a vu. On a choisi $\Omega=2 \pi \times 90$. On trouve alors $e=10 \mathrm{mV}$. Le bruit étant de l'ordre de $50 \mu \mathrm{W}$, le rapport signal sur bruit est de l'ordre de 200.

Cet appareillage a été testé avec différents supraconducteurs. Nous avons ainsi pu observer la transition, en faisant varier soit la température, soit le champ magnétique, de supraconducteurs de type II comme un alliage In-Tl de température critique $T_{\mathrm{c}}=3,25^{\circ} \mathrm{K}$ ou de supraconducteur de type I comme $\mathrm{Sn}\left(T_{\mathrm{c}}=3,74{ }^{\circ} \mathrm{K}\right), \mathrm{Zn}\left(T_{\mathrm{c}}=0,91^{\circ} \mathrm{K}\right), \mathrm{Cd}\left(T_{\mathrm{c}}=0,56^{\circ} \mathrm{K}\right)$ et $\mathrm{Ti}\left(T_{\mathrm{c}}=0,39^{\circ} \mathrm{K}\right)$. La figure 9 représente la transition du titane observée en fonction de la température.
Mais aucun échantillon de $\mathrm{C}_{8} \mathrm{~K}$ examiné n'a permis de mettre une transition en évidence, alors que si seule une fraction de l'échantillon devenait supraconductrice, la sensibilité de notre appareil suffirait à le détecter. Différents échantillons fabriqués à partir de graphites différents ont été essayés. Les échantillons fabriqués par nous-mêmes l'étaient à partir de graphite pyrolytique recuit à très haute température en

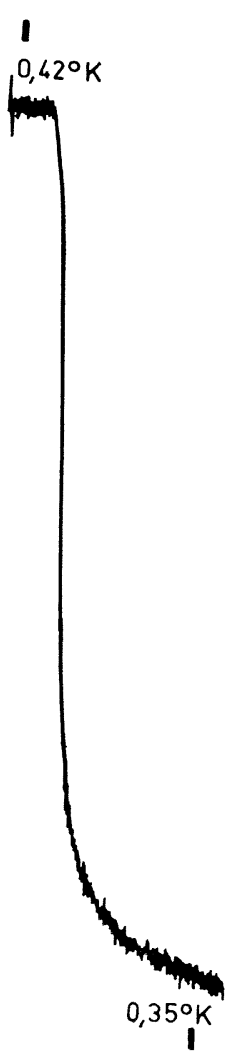

Fig. 9. - Enregistrement représentant la transition du titane.

provenance de Carbone Lorraine, ou de monocristaux naturels purifiés. Les échantillons fabriqués par le Dr K. A. Müller l'étaient à partir de graphite pyrolytique recuit en provenance de la Compagnie General Electric. Le graphite pyrolytique utilisé par le groupe de chercheurs qui a trouvé les composés de formule $\mathrm{C}_{8} \mathrm{M}$ supraconducteurs provenait de High Temperature Materials. Tous les échantillons que nous avons utilisés ont été examinés aux rayons $X$ qui ont permis de mettre en évidence les raies caractéristiques de la structure de $\mathrm{C}_{8} \mathrm{~K}$. Les raies correspondant au graphite disparaissent complètement montrant que le composé est homogène dans toute son épaisseur.

V. Conclusion. - L'étude de la variation de l'intensité du signal de résonance des électrons de conduction avec la température montre que cette variation correspond à la variation de la résistivité et donc que la susceptibilité magnétique de $\mathrm{C}_{8} \mathrm{~K}$ ne varie pas avec la température. D'autre part, nous n'avons pu mettre 
en évidence la transition à l'état supraconducteur dans aucun échantillon de $\mathrm{C}_{8} \mathrm{~K}$ refroidi jusqu'à $0,32^{\circ} \mathrm{K}$. Nous sommes donc en désaccord avec le groupe de chercheurs [10] qui a trouvé une température de transition pouvant varier de $0,39{ }^{\circ} \mathrm{K}$ à $0,55^{\circ} \mathrm{K}$ selon la stœchiométrie. Ceci est peut-être dû à la présence d'impuretés dans les matériaux de départ, potassium ou graphite, ou à la différence de qualité $\mathrm{du}$ graphite. Les propriétés physiques des graphites pyrolytiques varient beaucoup selon leur mode de préparation. De plus la température critique d'un supraconducteur varie exponentiellement avec la densité d'électrons au niveau de Fermi $N(0)$

$$
k T_{\mathrm{c}} \sim \exp -1 / N(0) V .
$$

Nos échantillons sont préparés en mettant le graphite en présence d'un excès de potassium et les dosages par pesée indiquent que leur composition est voisine de $\mathrm{C}_{7,94} \mathrm{~K}$ donc que le potassium est en léger excès. Il se peut cependant qu'ils aient une densité d'électrons plus faible que les échantillons étudiés par Hannay et Coll. [10] et donc une température de transition plus basse que celle que nous pouvions réaliser avec cet appareillage.

Remerciements. - Je tiens à remercier Monsieur le Professeur Abragam, Monsieur J. M. Winter et Monsieur K. A. Müller pour les discussions que j'ai eues avec eux au cours de ce travail. Je remercie également Monsieur Landesman et Monsieur Chapellier pour leurs observations sur l'obtention des basses températures, Madame Raynaud qui s'est chargée de la préparation des échantillons, Monsieur Jehanno de leur examen aux rayons x et Monsieur Delmas de la construction du cryostat.

\section{Bibliographie}

[1] Yosida (K.), Phys. Rev., 1958, 110, 769.

[2] Wright (F.), Phys. Rev., 1967, 163, 420.

[3] Schumacher (R. T.) et Slichter (C. P.), Phys. Rev., 1956, 101, 58.

[4] Kaplan (J. I.), Phys. Letters, 1965, 19, 226.

[5] De Gennes (P. G.), Note.

[6] Müller (K. A.), Note.

[7] Rudorff (W.) et Schultze (E.), Z. Anorg. Chem., 1954, 277, 156.

[8] Blackman (L. C. F.), Matthews (J. F.) et Ubbelohde (A. R.), Proc. Roy. Soc., 1960, A 258, 339.

[9] Müller (K. A.) et Kleiner (R.), Phys. Letters, 1962, $1,98$.

[10] Hannay (N. B.), Geballe (T. H.), Matthias (B. T.), Andres (K.), Schmidt (P.), NAIR (D. Mac), Phys. Rev. Letters, 1965, 14, 225.
[11] Roach (W. R.), Wheatley (J. C.) et Mota de VictoRIA (A. C.), R. S. I., 1964, 35, 634.

[12] Roberts (T. R.) et Sydoriak (S. G.), Phys. Rev., 1956, 102, 304.

[13] Wheatley (J. C.), Griffing (D. F.) et Estle (T. L.), R. S. I., 1956, 27, 1070.

[14] Anderson (A. C.), Reese (W.) et Wheatley (J. C.), R. S. I., 1963, 34, 1386.

[15] Hecht (S. I.) et Taub (J. J.), Microwaves, 1966, 5, 26.

[16] Teaney (D. T.), Klein (M. P.) et Portis (A. M.), R. S. I., 1961, 32, 721 .

[17] Feher (G.), Bell System Tech. J., 1957, 36, 449.

[18] Feher (G.) et Kip (A. F.), Phys. Rev., 1955, 98, 337.

[19] Tsoi (V.) et Sharvin (Yu V.), Communication privée. 